\title{
Application of A domian Decomposition method for Solving Fractional Differential Equation
}

\author{
Shayma Adil Murad Shaker Mahmood Rasheed \\ Dept. of Mathematics / College of Education \\ University of Dohuk
}

Received

29 / 04 / 2007
Accepted

$03 / 10 / 2007$

Keyword: fractional differential equation, Adomian polynomial, Adomian Decomposition method.

الملخص:

يتضمن هذا البحث تطبيق لطريقة ادومين التحليلية لحل المعادلة التفاضلية الكسرية:

$$
\text { (1.1) } \quad(n=1,2,3, \ldots) \quad n-1<\alpha<n, \quad A D^{m} y+B D^{\alpha} y+C y(x)=0
$$

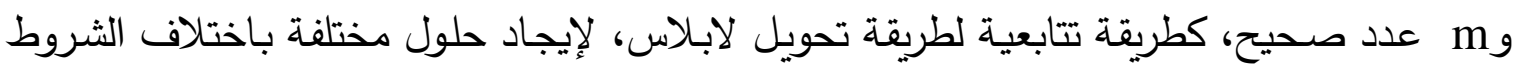

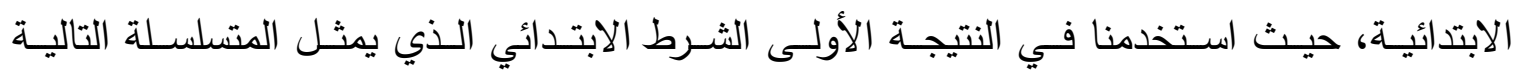
C $C_{1}, C_{2}, \ldots y(0)=\sum_{p=0}^{n-1} \frac{C_{p} x^{\alpha-p}}{\Gamma(\alpha-p+1)}$ النتيجة الثانية استخدمنا الثرط الابتدائي (0)

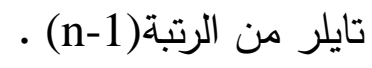

\section{Abstract}

In this paper we apply the Adomian decomposition method to find solution of fractional differential equation:

$$
A D^{m} y+B D^{\alpha} y+C y(x)=0 \quad, \quad n-1<\alpha<n \quad(n=1,2,3, \ldots)
$$

and $\mathrm{m}$ is integer number, with two different initial condition the first is $y(0)=\sum_{p=0}^{n-1} \frac{C_{p} x^{\alpha-p}}{\Gamma(\alpha-p+1)}$, where $\mathrm{C}_{1}, \mathrm{C}_{2, \ldots}$ are constant, the second initial condition $y(0)=\sum_{k=0}^{n-1} \frac{x^{k}}{k !} y^{(k)}(0)$ is the Taylor polynomial of order (n-1) for $\mathrm{y}$, as an alternative method of Laplace transform. 


\section{Introduction}

The decomposition method was introduced by Adomian [1] for solving linear and nonlinear functional equations (algebraic, differential, integral, integro differential equation) this method leads to computable, accurate, approximately convergent solutions to linear and nonlinear deterministic and stochastic operator equations. The solution can be verified to any degree of approximation. Elzaidi [5] solved the extraordinary differential equation $y^{\alpha}(x)=\frac{y}{x} \quad\left(x \neq 0, \alpha \in R^{+}\right) \quad$ With initial condition $\quad y_{0}(x)=\frac{c x^{\alpha-1}}{\Gamma(\alpha)}$ By using Laplace transform and Adomian decomposition method. This method simpler in computation. Diethelm and Neville [4] discussed existence and uniqueness theorem of differential equation of fractional order $D^{\alpha}\left(y-T_{n-1}[y]\right)(x)=f(x, y), n-1<\alpha \leq n$ where $T_{n-1}[y]$ is the Taylor polynomial of order (n-1) for $\mathrm{y}$, centered at 0 .

Luise [6] solved the fractional differential equation $D^{\alpha}\left(y-y_{0}\right)(x)=-\lambda^{\alpha} y(t), \quad n-1<\alpha \leq n$ with initial condition $y(0)=\sum_{k=0}^{n-1} \frac{x^{k}}{k !} y^{(k)}(0) \quad$ by using numerical method.

Saha and Bera [8] applied the Adomian decomposition method for the solution of fractional differential equation $\frac{d y}{d x}+\frac{d^{\frac{1}{2}} y}{d x^{\frac{1}{2}}}-2 y=0$, with initial condition $\mathrm{y}(0)=\mathrm{C}_{1}$

as on alternative method of Laplace transform.

\section{Definitions and Lemmas}

\section{Definition 2.1[7]}

The Gamma function is denoted by $\Gamma$ and is defined by the integral $\Gamma(\alpha)=\int_{0}^{\infty} e^{-x} x^{\alpha-1} d x \quad$,for $\quad \alpha>0$

\section{Definition 2.2[3]}

Let $\mathrm{f}$ a function which is defined a.e on [a,b].for $\alpha>0$, we define $I_{a}^{b} f=\frac{1}{\Gamma(\alpha)} \int_{a}^{b}(b-t)^{\alpha-1} f(t) d t$ provided that the integral (Lebsegue) exists. 


\section{Corollary 2.3[2]}

If $\alpha \in R$ and $\mathrm{f}(\mathrm{x})$ is defined a.e on $a \leq x \leq b$ we define $f_{(x)=\stackrel{x}{(\alpha)}-\alpha_{f}}$ for all $\mathrm{x} \in[\mathrm{a}, \mathrm{b}]$, provided that $\begin{gathered}x \\ \underset{a}{I}\end{gathered}-\alpha_{f}$ exists.

\section{Lemma 2.4[3]}

Let $\alpha, \beta \in R, \beta>-1$. If $x>a$ then

$$
{ }_{a}^{x}{ }_{a}^{\alpha-a)}{ }^{\beta}=\left\{\begin{array}{cc}
\frac{(x-a)^{\alpha+\beta} \Gamma(\beta+1)}{\Gamma(\alpha+\beta+1)} & \alpha+\beta \neq \text { negative integer } \\
0 & \alpha+\beta=\text { negative integer }
\end{array}\right.
$$

\section{Main results}

In this section we get some results about the solution of an extraordinary differential equation.

\section{Theorem(1)}

The extraordinary differential equation

$$
A D^{m} y+B D^{\alpha} y+C y(x)=0 \quad n-1<\alpha<n
$$

has a solution of the form $\mathrm{y}(\mathrm{x})=\mathrm{y}_{0}(\mathrm{x})+\mathrm{y}_{1}(\mathrm{x})+\mathrm{y}_{2}(\mathrm{x}) \ldots$, for the initial condition $y(a)=\sum_{p=0}^{n-1} \frac{C_{p}(x-a)^{\alpha-p}}{\Gamma(\alpha-p+1)}, \quad$ and $\quad y(0)=\sum_{p=0}^{n-1} \frac{C_{p} x^{\alpha-p}}{\Gamma(\alpha-p+1)}$, where $\mathrm{A}, \mathrm{B}, \mathrm{C}, \mathrm{C}_{0}, \mathrm{C}_{1}, \ldots$ are constant, and $\mathrm{m}$ is integer.

\section{Proof: (Adomain Decomposition method )}

Suppose that $L=\frac{d^{m}}{d x^{m}}$.

The Adomain decomposition method provide, we can write

$$
\begin{aligned}
& D^{m} y(x)=-\frac{1}{A}\left[B D^{\alpha} y+C y(x)\right] \\
& \frac{d^{m} y}{d x^{m}}=\frac{-1}{A}\left[B \frac{d^{\alpha} y}{d x^{\alpha}}+C y(x)\right]
\end{aligned}
$$

operate equation (3.1.2) by $L^{-1}$ getting 


$$
\begin{aligned}
& y(x)=-\frac{1}{A}\left[B L^{-1}\left(\frac{d^{\alpha} y}{d x^{\alpha}}\right)+C L^{-1}(y)\right] \text { this implies that } \\
& y(x)=-\frac{1}{A}\left[C \frac{d^{-m} y}{d x^{-m}}+B \frac{d^{\alpha-m} y}{d x^{\alpha-m}}\right]
\end{aligned}
$$

In the light of the Adomian decomposition method, we assume $\mathrm{y}(\mathrm{x})=\mathrm{y}_{0}(\mathrm{x})+\mathrm{y}_{1}(\mathrm{x})+\mathrm{y}_{2}(\mathrm{x}) \ldots$

To be the solution of equation (3.1.1), where

$$
y_{1}(x)=-\frac{1}{A}\left[C \frac{d^{-m} y_{0}}{d x^{-m}}+B \frac{d^{\alpha-m} y_{0}}{d x^{\alpha-m}}\right]
$$

by using Corollary (2.3) we get

$$
\begin{aligned}
& y_{1}(x)=-\frac{1}{A}\left[\begin{array}{c}
x \\
C \stackrel{x}{I} \\
0
\end{array} y_{0}(t)+B \underset{0}{I} m-\alpha y_{0}(t)\right]
\end{aligned}
$$

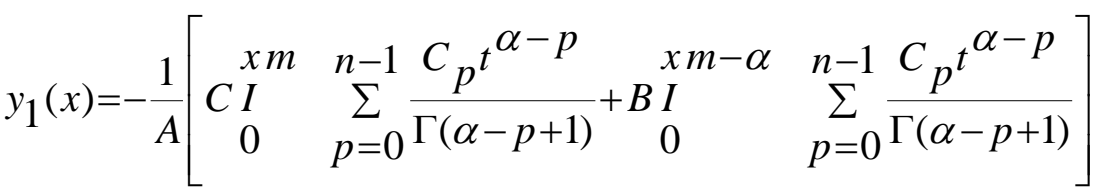

by using lemma(2.4) we get

$$
\begin{aligned}
& y_{1}(x)=-\frac{1}{A}\left[C \sum_{p=0}^{n-1} \frac{C_{p} x^{\alpha-p+m}}{\Gamma(\alpha-p+m+1)}+B \sum_{p=0}^{n-1} \frac{C_{p} x^{m-p}}{\Gamma(m-p+1)}\right] \\
& y_{2}(x)=-\frac{1}{A}\left[C \frac{d^{-m} y_{1}}{d x^{-m}}+B \frac{d^{\alpha-m} y_{1}}{d x^{\alpha-m}}\right]
\end{aligned}
$$

by using Corollary (2.3) we get

$$
\begin{aligned}
& y_{2}(x)=-\frac{1}{A}\left[\begin{array}{ccc}
x & & x \\
C \stackrel{I}{I}_{0}^{m} & y_{1}+B \stackrel{0}{I}^{m-\alpha} y_{1}
\end{array}\right]
\end{aligned}
$$

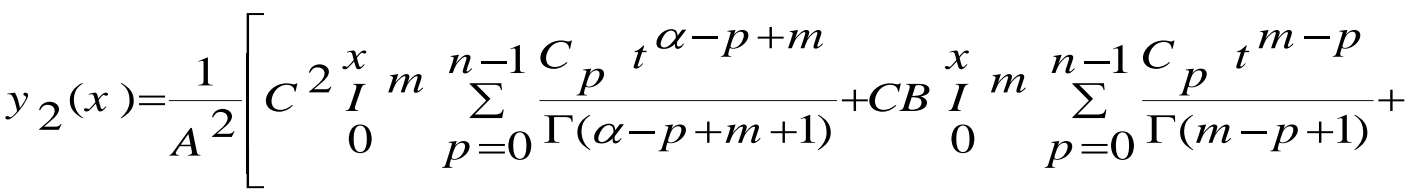

$$
\begin{aligned}
& \left.+B C \underset{0}{I} \underset{p=0}{m-\alpha} \sum_{p=0}^{n-1} \frac{C_{p} t^{\alpha-p+m}}{\Gamma(\alpha-p+m+1)}+B{ }_{0}^{2} I_{p=0}^{x} \sum_{p=0}^{n-1} \frac{C_{p} t^{m-p}}{\Gamma(m-p+1)}\right]
\end{aligned}
$$

by using lemma (2.4) 


$$
\begin{aligned}
& y_{2}(x)=\frac{1}{A^{2}}\left[C^{2} \sum_{p=0}^{n-1} \frac{C p^{x-p+2 m}}{\Gamma(\alpha-p+2 m+1)}+2 C B \sum_{p=0}^{n-1} \frac{C_{p} x^{2 m-p}}{\Gamma(2 m-p+1)}\right. \\
& \left.+B_{p=0}^{2} \sum_{p=1}^{n-1} \frac{C_{p}^{2 m-\alpha-p}}{\Gamma(2 m-\alpha-p+1)}\right] \\
& y_{3}(x)=-\frac{1}{A}\left[C \frac{d^{-m} y_{2}}{d x^{-m}}+B \frac{d^{\alpha-m} y_{2}}{d x^{\alpha-m}}\right]
\end{aligned}
$$

by using the Corollary (2.3) we get

$$
y_{3}(x)=-\frac{1}{A}\left[\begin{array}{c}
{ }_{C}^{x} \\
0
\end{array} y_{2}+B{\underset{0}{I}}^{x} m-\alpha y_{2}\right]
$$

by using lemma(2.4)

$$
\begin{aligned}
& y_{3}(x)=-\frac{1}{A^{3}}\left[C^{3} \sum_{p=0}^{n-1} \frac{C_{p} x^{\alpha-p+3 m}}{\Gamma(\alpha-p+3 m+1)}+3 C^{2} B \sum_{p=0}^{n-1} \frac{C_{p} x^{3 m-p}}{\Gamma(3 m-p+1)}+\right. \\
& \left.+3 B{ }^{2} C \sum_{p=0}^{n-1} \frac{C_{p} x^{3 m-\alpha-p}}{\Gamma(3 m-\alpha-p+1)}+B \sum_{p=0}^{3-1} \frac{C_{p} x^{3 m-2 \alpha-p}}{\Gamma(3 m-2 \alpha-p+1)}\right] \\
& y_{4}(x)=\frac{1}{A^{4}}\left[C^{4} \sum_{p=0}^{n-1} \frac{C_{p} x^{\alpha-p+4 m}}{\Gamma(\alpha-p+4 m+1)}+4 C^{3} B \sum_{p=0}^{n-1} \frac{C_{p} x^{4 m-p}}{\Gamma(4 m-p+1)}+\right. \\
& \left.+6 B^{2} C^{2} \sum_{p=0}^{n-1} \frac{C_{p} x^{4 m-\alpha-p}}{\Gamma(4 m-\alpha-p+1)}+4 C B \sum_{p=0}^{3-1} \frac{C_{p} x^{4 m-2 \alpha-p}}{\Gamma(4 m-2 \alpha-p+1)}+B \sum_{p=0}^{4-1} \frac{C_{p} x^{4 m-3 \alpha-p}}{\Gamma(4 m-3 \alpha-p+1)}\right] \\
& y_{5}(x)=\frac{-1}{A^{5}}\left[C^{5} \sum_{p=0}^{n-1} \frac{C_{p} x^{\alpha-p+5 m}}{\Gamma(\alpha-p+5 m+1)}+5 C^{4} B \sum_{p=0}^{n-1} \frac{C_{p} x^{5 m-p}}{\Gamma(5 m-p+1)}+\right. \\
& +10 B^{2} C^{3} \sum_{p=0}^{n-1} \frac{C_{p} x^{5 m-\alpha-p}}{\Gamma(5 m-\alpha-p+1)}+10 C^{2} B \sum_{p=0}^{3 \sum_{1}^{n-1}} \frac{C_{p} x^{5 m-2 \alpha-p}}{\Gamma(5 m-2 \alpha-p+1)}+5 C B \sum_{p=0}^{4 \sum_{n}^{n-1}} \frac{C_{p} x^{5 m-3 \alpha-p}}{\Gamma(5 m-3 \alpha-p+1)}+ \\
& \left.+B \sum_{p=0}^{n-1} \frac{C_{p} x^{5 m-4 \alpha-p}}{\Gamma(5 m-4 \alpha-p+1)}\right]
\end{aligned}
$$

and so on. Therefore the solution of (3.1.1) is $y(x)=y_{0}(x)+y_{1}(x)+y_{2}(x)+\ldots$ 


\section{Application of A domian Decomposition method for Solving Fractional ...}

$$
\begin{aligned}
& y(x)=\sum_{p=0}^{n-1} \frac{C_{p} x^{\alpha-p}}{\Gamma(\alpha-p+1)}-\frac{1}{A}\left[C \sum_{p=0}^{n-1} \frac{C_{p} x^{\alpha-p+m}}{\Gamma(\alpha-p+m+1)}+B \sum_{p=0}^{n-1} \frac{C_{p} x^{m-p}}{\Gamma(m-p+1)}\right]+
\end{aligned}
$$

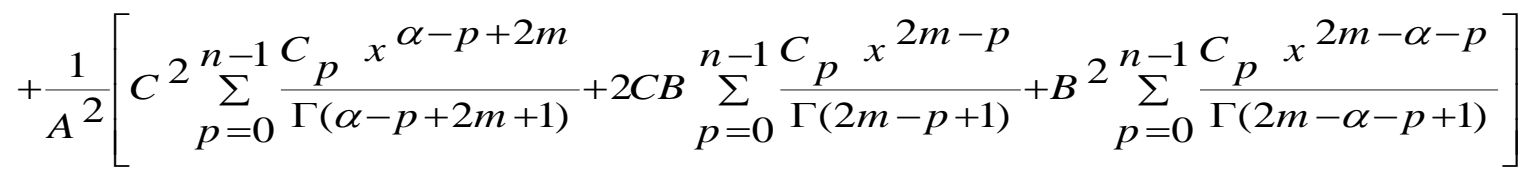

$$
\begin{aligned}
& -\frac{1}{A^{3}}\left[C^{3} \sum_{p=0}^{n-1} \frac{C_{p} x^{\alpha-p+3 m}}{\Gamma(\alpha-p+3 m+1)}+3 C^{2} B \sum_{p=0}^{n-1} \frac{C_{p} x^{3 m-p}}{\Gamma(3 m-p+1)}+3 B{ }^{2} C \sum_{p=0}^{n-1} \frac{C_{p} x^{3 m-\alpha-p}}{\Gamma(3 m-\alpha-p+1)}+\right. \\
& \left.+B \sum_{p=0}^{3-1} \frac{C_{p} x^{3 m-2 \alpha-p}}{\Gamma(3 m-2 \alpha-p+1)}\right]+\frac{1}{A^{4}}\left[C^{4} \sum_{p=0}^{n-1} \frac{C_{p} x^{\alpha-p+4 m}}{\Gamma(\alpha-p+4 m+1)}+4 C^{3} B \sum_{p=0}^{n-1} \frac{C_{p} x^{4 m-p}}{\Gamma(4 m-p+1)}+\right. \\
& \left.+6 B^{2} C^{2} \sum_{p=0}^{n-1} \frac{C_{p} x^{4 m-\alpha-p}}{\Gamma(4 m-\alpha-p+1)}+4 C B \sum_{p=0}^{3-1} \frac{C_{p} x^{4 m-2 \alpha-p}}{\Gamma(4 m-2 \alpha-p+1)}+B \sum_{p=0}^{4-1} \frac{C_{p} x^{4 m-3 \alpha-p}}{\Gamma(4 m-3 \alpha-p+1)}\right]- \\
& -\frac{1}{A^{5}}\left[C^{5} \sum_{p=0}^{n-1} \frac{C_{p^{x}} x^{\alpha-p+5 m}}{\Gamma(\alpha-p+5 m+1)}+5 C^{4} B \sum_{p=0}^{n-1} \frac{C_{p^{2}} x^{5 m-p}}{\Gamma(5 m-p+1)}+10 B^{2} C^{3} \sum_{p=0}^{n-1} \frac{C_{p} x^{5 m-\alpha-p}}{\Gamma(5 m-\alpha-p+1)}+\right. \\
& \left.+10 C^{2} B \sum_{p=0}^{3-1} \frac{C_{p} x^{5 m-2 \alpha-p}}{\Gamma(5 m-2 \alpha-p+1)}+5 C B \sum_{p=0}^{4-1} \frac{C_{p} x^{5 m-3 \alpha-p}}{\Gamma(5 m-3 \alpha-p+1)}+B \sum_{p=0}^{5 \sum^{n-1} \frac{C_{p} x^{5 m-4 \alpha-p}}{\Gamma(5 m-4 \alpha-p+1)}}\right] \\
& +\ldots
\end{aligned}
$$

Example: To illustrate the procedure

$$
\text { let } D^{2} y(x)+D^{\frac{3}{2}} y(x)+y(x)=0 \quad 1<\alpha<2
$$

by using theorem (1) we get

$$
\begin{aligned}
& y(x)= \sum_{p=0}^{1} \frac{C_{p} x^{\frac{3}{2}-p}}{\Gamma\left(\frac{5}{2}-p\right)}-\left(\sum_{p=0}^{1} \frac{C_{p} x^{\frac{7}{2}-p}}{\Gamma\left(\frac{9}{2}-p\right)}+\sum_{p=0}^{1} \frac{C_{p} x^{2-p}}{\Gamma(3-p)}\right)+\sum_{p=0}^{1} \frac{C_{p} x^{\frac{11}{2}-p}}{\Gamma\left(\frac{13}{2}-p\right)}+2 \sum_{p=0}^{1} \frac{C_{p} x^{4-p}}{\Gamma(5-p)}+\sum_{p=0}^{1} \frac{C_{p} x^{\frac{5}{2}-p}}{\Gamma\left(\frac{7}{2}-p\right)}- \\
&-\left(\sum_{p=0}^{1} \frac{C_{p} x^{\frac{15}{2}-p}}{\Gamma\left(\frac{17}{2}-p\right)}+3 \sum_{p=0}^{1} \frac{C_{p} x^{6-p}}{\Gamma(7-p)}+3 \sum_{p=0}^{1} \frac{C_{p} x^{\frac{9}{2}-p}}{\Gamma\left(\frac{11}{2}-p\right)}+\sum_{p=0}^{1} \frac{C_{p} x^{3-p}}{\Gamma(4-p)}\right)+ \\
&+\sum_{p=0}^{1} \frac{C_{p} x^{\frac{19}{2}-p}}{\Gamma\left(\frac{21}{2}-p\right)}+4 \sum_{p=0}^{1} \frac{C_{p} x^{8-p}}{\Gamma(9-p)}+6 \sum_{p=0}^{1} \frac{C_{p} x^{\frac{13}{2}-p}}{\Gamma\left(\frac{15}{2}-p\right)}+4 \sum_{p=0}^{1} \frac{C_{p} x^{5-p}}{\Gamma(6-p)}+\sum_{p=0}^{1} \frac{C_{p} x^{\frac{7}{2}-p}}{\Gamma\left(\frac{9}{2}-p\right)}
\end{aligned}
$$




$$
\begin{aligned}
& y(x)=\frac{C_{0} x^{\frac{3}{2}}}{\Gamma\left(\frac{5}{2}\right)}-\frac{C_{0} x^{2}}{\Gamma(3)}+\frac{C_{0} x^{\frac{5}{2}}}{\Gamma\left(\frac{7}{2}\right)}-\frac{C_{0} x^{3}}{\Gamma(4)}-\frac{C_{0} x^{\frac{7}{2}}}{\Gamma\left(\frac{9}{2}\right)}+2 \frac{C_{0} x^{4}}{\Gamma(5)}-3 \frac{C_{0} x^{\frac{9}{2}}}{\Gamma\left(\frac{11}{2}\right)}+\frac{C_{0} x^{\frac{11}{2}}}{\Gamma\left(\frac{13}{2}\right)}-3 \frac{C_{0} x^{6}}{\Gamma(7)}-\frac{C_{0} x^{\frac{15}{2}}}{\Gamma\left(\frac{17}{2}\right)}+\frac{C_{0} x^{\frac{17}{2}}}{\Gamma\left(\frac{19}{2}\right)} \\
& \frac{C_{1} x^{\frac{1}{2}}}{\Gamma\left(\frac{3}{2}\right)}-\frac{C_{1} x}{\Gamma(2)}+\frac{C_{1} x^{\frac{3}{2}}}{\Gamma\left(\frac{5}{2}\right)}-\frac{C_{1} x^{2}}{\Gamma(3)}-\frac{C_{1} x^{\frac{5}{2}}}{\Gamma\left(\frac{7}{2}\right)}+2 \frac{C_{1} x^{3}}{\Gamma(4)}+\frac{C_{1} x^{\frac{9}{2}}}{\Gamma\left(\frac{11}{2}\right)}-\frac{C_{1} x^{\frac{13}{2}}}{\Gamma\left(\frac{15}{2}\right)}+\frac{C_{1} x^{\frac{17}{2}}}{\Gamma\left(\frac{19}{2}\right)}+\ldots
\end{aligned}
$$

\section{Theorem(2)}

The extraordinary differential equation:

$$
A D^{m} y+B D^{\alpha} y+C y(x)=0 \quad n-1<\alpha<n
$$

has a solution of the form $\mathrm{y}(\mathrm{x})=\mathrm{y}_{0}(\mathrm{x})+\mathrm{y}_{1}(\mathrm{x})+\mathrm{y}_{2}(\mathrm{x}) \ldots$, for the initial condition $y(0)=\sum_{k=0}^{n-1} \frac{x^{k}}{k !} y^{(k)}(0)$ which is a Taylor polynomial of order (n-1) for $\mathrm{y}$, centered at 0 , where $\mathrm{A}, \mathrm{B}, \mathrm{C}$ are constant and $\mathrm{m}$ is integer.

\section{Proof: (Adomain Decomposition method)}

Suppose that $L=\frac{d^{m}}{d x^{m}}$.

using the Adomian decomposition method, we can write

$$
\begin{aligned}
& D^{m} y(x)=-\frac{1}{A}\left[B D^{\alpha} y+C y(x)\right] \\
& \frac{d^{m} y}{d x^{m}}=\frac{-1}{A}\left[B \frac{d^{\alpha} y}{d x^{\alpha}}+C y(x)\right]
\end{aligned}
$$

Operating equation (3.2.2) by $L^{-1}$ we get

$$
\begin{aligned}
& y(x)=-\frac{1}{A}\left[B L^{-1}\left(\frac{d^{\alpha} y}{d x^{\alpha}}\right)+C L^{-1}(y)\right] \text { this implies that } \\
& y(x)=-\frac{1}{A}\left[C \frac{d^{-m} y}{d x^{-m}}+B \frac{d^{\alpha-m} y}{d x^{\alpha-m}}\right]
\end{aligned}
$$

In the light of the Adomian decomposition method, we assume $\mathrm{y}(\mathrm{x})=\mathrm{y}_{0}(\mathrm{x})+\mathrm{y}_{1}(\mathrm{x})+\mathrm{y}_{2}(\mathrm{x}) \ldots$

To be the solution of equation (3.2.1), where

$$
y_{1}(x)=-\frac{1}{A}\left[C \frac{d^{-m} y_{0}}{d x^{-m}}+B \frac{d^{\alpha-m} y_{0}}{d x^{\alpha-m}}\right]
$$

by using Corollary (2.3) we get 


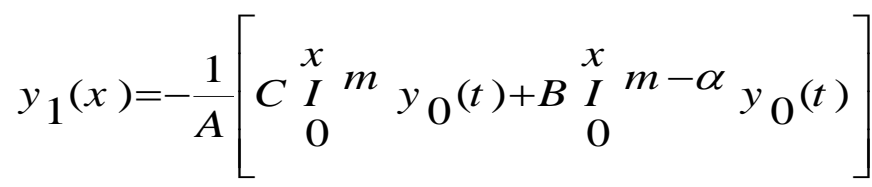

$$
\begin{aligned}
& y_{1}(x)=-\frac{1}{A}\left[\begin{array}{c}
x \\
C \\
0
\end{array} \sum_{k=0}^{n-1} \frac{{ }_{t}^{k}}{\Gamma(k+1)} y^{(k)}(0)+B{\underset{0}{I}}_{k=0}^{x} \sum_{k=\alpha}^{n-1} \frac{t^{k}}{\Gamma(k+1)} y^{(k)}(0)\right]
\end{aligned}
$$

by using lemma(2.4) we get

$$
\begin{aligned}
& y_{1}(x)=-\frac{1}{A}\left[C \sum_{k=0}^{n-1} \frac{x^{m+k}}{\Gamma(m+k+1)} y(k)(0)+B \sum_{k=0}^{n-1} \frac{x^{m-\alpha+k}}{\Gamma(m-\alpha+k+1)} y(k)(0)\right] \\
& y_{2}(x)=-\frac{1}{A}\left[C \frac{d^{-m} y_{1}}{d x^{-m}}+B \frac{d^{\alpha-m} y_{1}}{d x}\right]
\end{aligned}
$$

by using Corollary (2.3) we get:

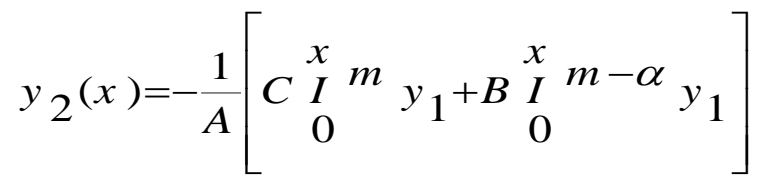

$$
\begin{aligned}
& y_{2}(x)=\frac{1}{A^{2}}\left[C^{2} \underset{I}{I_{0}^{m}} \sum_{k=0}^{n-1} \frac{t^{m+k}}{\Gamma(m+k+1)} y(k)(0)+C B \underset{0}{I} \sum_{k=0}^{x} \frac{t^{m-\alpha+k}}{\Gamma(m-\alpha+k+1)} y(k)(0)+\right. \\
& \left.+B C I_{0}^{x} m \sum_{k=0}^{m-\alpha} \frac{t^{m+k}}{\Gamma(m+k+1)} y(k)(0)+B{ }_{0}^{2}{ }_{I}^{x} \sum_{k=0}^{m-\alpha} \frac{t^{m-\alpha+k}}{\Gamma(m-\alpha+k+1)} y^{(k)}(0)\right]
\end{aligned}
$$

by using lemma (2.4)

$$
\begin{aligned}
& y_{2}(x)=\frac{1}{A^{2}}\left[C^{2} \sum_{k=0}^{n-1} \frac{x^{2 m+k}}{\Gamma(2 m+k+1)} y(k)(0)+2 C B \sum_{k=0}^{n-1} \frac{x^{2 m-\alpha+k}}{\Gamma(2 m-\alpha+k+1)} y(k)(0)\right. \\
& \left.+B \sum_{k=0}^{n-1} \frac{x^{2 m-2 \alpha+k}}{\Gamma(2 m-2 \alpha+k+1)} y^{(k)}(0)\right] \\
& y_{3}(x)=-\frac{1}{A}\left[C \frac{d^{-m} y_{2}}{d x^{-m}}+B \frac{d^{\alpha-m} y_{2}}{d x^{\alpha-m}}\right] \\
& y_{3}(x)=-\frac{1}{A^{3}}\left[C^{3} \sum_{k=0}^{n-1} \frac{x^{3 m+k}}{\Gamma(3 m+k+1)} y(k)(0)+3 C^{2} B \sum_{k=0}^{n-1} \frac{x^{3 m-\alpha+k}}{\Gamma(3 m-\alpha+k+1)} y(k)(0)+\right. \\
& \left.3 B^{2} C \sum_{k=0}^{n-1} \frac{x^{3 m-2 \alpha+k}}{\Gamma(3 m-2 \alpha+k+1)} y^{(k)}(0)+B \sum_{k=0}^{3-1} \frac{x^{3 m-3 \alpha+k}}{\Gamma(3 m-3 \alpha+k+1)} y^{(k)}(0)\right]
\end{aligned}
$$




\section{Application of A domian Decomposition method for Solving Fractional ...}

$$
\begin{aligned}
& y_{4}(x)=\frac{1}{A^{4}}\left[C^{4} \sum_{k=0}^{n-1} \frac{x^{4 m+k}}{\Gamma(4 m+k+1)} y^{(k)}(0)+4 C^{3} B \sum_{k=0}^{n-1} \frac{x^{4 m-\alpha+k}}{\Gamma(4 m-\alpha+k+1)} y(k)(0)+\right. \\
& 6 B^{2} C^{2} \sum_{k=0}^{n-1} \frac{x^{4 m-2 \alpha+k}}{\Gamma(4 m-2 \alpha+k+1)} y{ }^{(k)}(0)+4 C B^{3} \sum_{k=0}^{n-1} \frac{x^{4 m-3 \alpha+k}}{\Gamma(4 m-3 \alpha+k+1)} y^{(k)}(0) \\
& \left.+B \sum_{k=0}^{n-1} \frac{x^{4 m-4 \alpha+k}}{\Gamma(4 m-4 \alpha+k+1)} y^{(k)}(0)\right] \\
& y_{5}(x)=\frac{-1}{A^{5}}\left[C^{5} \sum_{k=0}^{n-1} \frac{x^{5 m+k}}{\Gamma(5 m+k+1)} y^{(k)}(0)+5 C{ }^{4} B \sum_{k=0}^{n-1} \frac{x^{5 m-\alpha+k}}{\Gamma(5 m-\alpha+k+1)} y(k)(0)+\right. \\
& 10 B^{2} C^{3} \sum_{k=0}^{n-1} \frac{x^{5 m-2 \alpha+k}}{\Gamma(5 m-2 \alpha+k+1)} y{ }^{(k)}(0)+10 C^{2} B^{3} \sum_{k=0}^{n-1} \frac{x^{5 m-3 \alpha+k}}{\Gamma(5 m-3 \alpha+k+1)} y^{(k)}(0)+ \\
& \left.5 C B \sum_{k=0}^{n-1} \frac{x^{5 m-4 \alpha+k}}{\Gamma(5 m-4 \alpha+k+1)} y^{(k)}(0)+B \sum_{k=0}^{5-1} \frac{x^{5 m-5 \alpha+k}}{\Gamma(5 m-5 \alpha+k+1)} y^{(k)}(0)\right]
\end{aligned}
$$

and so on. Therefore the solution of $(3.2 .1)$ is $y(x)=y_{0}(x)+y_{1}(x)+y_{2}(x)+\ldots$

$$
\begin{aligned}
& y(x)=\sum_{k=0}^{n-1} \frac{x^{k}}{k !} y^{(k)}(0)-\frac{1}{A}\left[C \sum_{k=0}^{n-1} \frac{x^{m+k}}{\Gamma(m+k+1)} y^{(k)}(0)+B \sum_{k=0}^{n-1} \frac{x^{m-\alpha+k}}{\Gamma(m-\alpha+k+1)} y^{(k)}(0)\right]+ \\
& \frac{1}{A^{2}}\left[C^{2} \sum_{k=0}^{n-1} \frac{x^{2 m+k}}{\Gamma(2 m+k+1)} y^{(k)}(0)+2 C B \sum_{k=0}^{n-1} \frac{x^{2 m-\alpha+k}}{\Gamma(2 m-\alpha+k+1)} y^{(k)}(0)\right. \\
& \left.+B \sum_{k=0}^{2} \frac{x^{2 m-2 \alpha+k}}{\Gamma(2 m-2 \alpha+k+1)} y^{(k)}(0)\right]-\frac{1}{A^{3}}\left[C^{3} \sum_{k=0}^{n-1} \frac{x^{3 m+k}}{\Gamma(3 m+k+1)} y^{(k)}(0)+3 C^{2} B \sum_{k=0}^{n-1} \frac{x^{3 m-\alpha+k}}{\Gamma(3 m-\alpha+k+1)} y{ }^{(k)}(0)\right. \\
& +\frac{1}{A^{4}}\left[C^{4} \sum_{k=0}^{n-1} \frac{x^{4 m+k}}{\Gamma(4 m+k+1)} y(k)(0)+4 C^{3} B \sum_{k=0}^{n-1} \frac{x^{4 m-\alpha+k}}{\Gamma(4 m-\alpha+k+1)} y(k)(0)+\right. \\
& +6 B^{2} C^{2} \sum_{k=0}^{n-1} \frac{x^{4 m-2 \alpha+k}}{\Gamma(4 m-2 \alpha+k+1)} y^{(k)}(0)+4 C B \sum_{k=0}^{3-1} \frac{x^{4 m-3 \alpha+k}}{\Gamma(4 m-3 \alpha+k+1)} y^{(k)}(0) \\
& \left.+B \sum_{k=0}^{n-1} \frac{x^{4 m-4 \alpha+k}}{\Gamma(4 m-4 \alpha+k+1)} y^{(k)}(0)\right]-\frac{1}{A^{5}}\left[C^{5} \sum_{k=0}^{n-1} \frac{x^{5 m+k}}{\Gamma(5 m+k+1)} y^{(k)}(0)\right. \\
& +5 C^{4} B \sum_{k=0}^{n-1} \frac{x^{5 m-\alpha+k}}{\Gamma(5 m-\alpha+k+1)} y(k)(0)+10 B^{2} C^{3} \sum_{k=0}^{n-1} \frac{x^{5 m-2 \alpha+k}}{\Gamma(5 m-2 \alpha+k+1)} y^{(k)}(0)+ \\
& +10 C^{2} B \sum_{k=0}^{n-1} \frac{x^{5 m-3 \alpha+k}}{\Gamma(5 m-3 \alpha+k+1)} y^{(k)}(0)+5 C B \sum_{k=0}^{4-1} \frac{x^{5 m-4 \alpha+k}}{\Gamma(5 m-4 \alpha+k+1)} y(k)(0)
\end{aligned}
$$




$$
\left.+B \sum_{k=0}^{5-1} \frac{x^{5 m-5 \alpha+k}}{\Gamma(5 m-5 \alpha+k+1)} y^{(k)}(0)\right]+\ldots
$$

Example: To illustrate the procedure

$$
\begin{array}{ll}
\text { let } \quad D y(x)+D^{\frac{3}{2}} y(x)+y(x)=0 & 1<\alpha<2 \\
y(0)=\sum_{k=0}^{1} \frac{x^{k}}{k !} y^{(k)}(0)=y^{(0)}(0)+x y^{(1)}(0) & \\
y^{(0)}(0)=C_{0} \quad, \quad y^{(1)}(0)=C_{1} &
\end{array}
$$

by using theorem (2) we get

$$
\begin{aligned}
& y(x)=\sum_{k=0}^{1} \frac{x^{k}}{k !} y^{(k)}(0)-\left(\sum_{k=0}^{1} \frac{x^{1+k}}{\Gamma(k+2)} y^{(k)}(0)+\sum_{k=0}^{1} \frac{x^{k-\frac{1}{2}}}{\Gamma\left(k+\frac{1}{2}\right)} y^{(k)}(0)+\sum_{k=0}^{1} \frac{x^{2+k}}{\Gamma(3+k)} y^{(k)}(0)\right. \\
& +2 C B \sum_{k=0}^{1} \frac{x^{k+\frac{1}{2}}}{\Gamma\left(k+\frac{3}{2}\right)} y^{(k)}(0)+\sum_{k=0}^{1} \frac{x^{k-1}}{\Gamma(k)} y^{(k)}(0)-\sum_{k=0}^{1} \frac{x^{3+k}}{\Gamma(4+k)} y^{(k)}(0)-3 \sum_{k=0}^{1} \frac{x^{\frac{3}{2}+k}}{\Gamma\left(k+\frac{5}{2}\right)} y^{(k)}(0) \\
& -3 \sum_{k=0}^{1} \frac{x^{k}}{\Gamma(k+1)} y^{(k)}(0)-\sum_{k=0}^{1} \frac{x^{k-\frac{3}{2}}}{\Gamma\left(k-\frac{1}{2}\right)} y^{(k)}(0) \quad+\ldots
\end{aligned}
$$

Provided ( $\mathrm{k}-1 \neq$ negative integer number)

$$
\begin{aligned}
& y(x)=\left(C_{0}+C_{1} x\right)-\left(C_{0} x+C_{1} \frac{x^{2}}{\Gamma(3)}\right)-\left(C_{0} \frac{x^{-\frac{1}{2}}}{\Gamma\left(\frac{1}{2}\right)}+C_{1} \frac{x^{\frac{1}{2}}}{\Gamma\left(\frac{3}{2}\right)}\right)+\left(C_{0} \frac{x^{2}}{\Gamma(3)}+C_{1} \frac{x^{3}}{\Gamma(4)}\right)+2\left(C_{0} \frac{x^{\frac{1}{2}}}{\Gamma\left(\frac{3}{2}\right)}+C_{1} \frac{x^{\frac{3}{2}}}{\Gamma\left(\frac{5}{2}\right)}\right) \\
& +\left(C_{0} x^{-1}+C_{1}\right)-\left(C_{0} \frac{x^{3}}{\Gamma(4)}+C_{1} \frac{x^{4}}{\Gamma(5)}\right)-3\left(C_{0} \frac{x^{\frac{3}{2}}}{\Gamma\left(\frac{5}{2}\right)}+C_{1} \frac{x^{\frac{5}{2}}}{\Gamma\left(\frac{7}{2}\right)}\right)-3\left(C_{0}+C_{1} x\right)-\left(C_{0} \frac{x^{-\frac{3}{2}}}{\Gamma\left(-\frac{1}{2}\right)}+C_{1} \frac{x^{-\frac{1}{2}}}{\Gamma\left(\frac{1}{2}\right)}\right)+\ldots
\end{aligned}
$$

by using lemma(2.4) we get $\left(C_{0} x^{-1}=0\right)$, there for the solution is

$$
\begin{gathered}
y(x)=-C_{0} \frac{x^{-\frac{3}{2}}}{\Gamma\left(-\frac{1}{2}\right)}-C_{0} \frac{x^{-\frac{1}{2}}}{\Gamma\left(\frac{1}{2}\right)}-2 C_{0}+2 C_{0} \frac{x^{\frac{1}{2}}}{\Gamma\left(\frac{3}{2}\right)}-C_{0} x-3 C_{0} \frac{x^{\frac{3}{2}}}{\Gamma\left(\frac{5}{2}\right)}+C_{0} \frac{x^{2}}{\Gamma(3)}+-C_{0} \frac{x^{3}}{\Gamma(4)} \\
-C_{1} \frac{x^{-\frac{1}{2}}}{\Gamma\left(\frac{1}{2}\right)}+C_{1}-C_{1} \frac{x^{\frac{1}{2}}}{\Gamma\left(\frac{3}{2}\right)}-2 C_{1} x+2 C_{1} \frac{x^{\frac{3}{2}}}{\Gamma\left(\frac{5}{2}\right)}-C_{1} \frac{x^{2}}{\Gamma(3)}-3 C_{1} \frac{x^{\frac{5}{2}}}{\Gamma\left(\frac{7}{2}\right)}+C_{1} \frac{x^{3}}{\Gamma(4)}-C_{1} \frac{x^{4}}{\Gamma(5)}+\ldots
\end{gathered}
$$




\section{References}

1) Adomian, G., Nonlinear Stochastic Operator Equation, Academic press, New York, NY, (1986).

2) Al-Bassam, M. A., Some existence theorem on differential equation of generalized order, Canada, Math, 10 (1964).

3) Barrett, J. H., Differential equations of non-integer order, Can. J. Math, 6(1954), pp529-541.

4) Diethelm, K. and Neville, J. Analysis of Fractional Differential Equations, J. Math. Anal. Appl., 265, ( 2002) pp. 229-248,.",

5) El-zaidi, S. M., On the solution of the differential equation, Journal of Fractional Calculus, 11(1997), pp75-80.

6) Luise, B. Numerical Treatment of Differential Equation of fractional order, Non linear World 4, (1997), pp473-490.

7) Oldham, K. B. and j. Spanier, The Fractional Calculus, Academic press, New York, London, (1974), pp47.

8) Saha, R. S. and Bera, R. k., Solution of an extraordinary differential equation. By Adomian Decomposition method, Journal of Applied mathematics, 4(2004), pp331-338. 